\title{
Time to benefit for colorectal cancer screening: survival meta-analysis of flexible sigmoidoscopy trials
}

The second sentence in the paragraph on "Comparison with other studies" should not be linked to reference 30 (BMJ 2015;350:h1662, doi:10.1136/bmj.h1662). This sentence, "Our previous survival meta-analysis of the time to benefit for fecal occult blood testing suggested that it would take 10 years $(95 \%$ confidence interval 6 to 16) to avoid one death from colorectal cancer per 1000 people screened," is, in fact, supported by the study cited in reference 22 . Reference 30 shouldn't be a part of the references and its inclusion is an error.

Cite this as: BMJ 2015;350:h2228

๑ BMJ Publishing Group Ltd 2015 